\title{
Die Ausbildung medizinischer Gutachter in der Schweiz - bisher und zukünftig
}

Hans Rudolf Stöckli, Leiter Gutachterkurse SIM

Korrespondenz:

Dr. med. Hans Rudolf Stöckli

Neurologie FMH

Kasernenstrasse 22a

$\mathrm{CH}-4410$ Liestal

hansrudolf.stoeckli@hin.ch
Die Forderung nach medizinischen Gutachten zwecks Festlegung der Arbeitsfähigkeit, der zumutbaren Arbeitsleistung, der medizinischtheoretischen Invalidität, der Integritätsentschädigung, der Kausalität sowie der Abgrenzung Unfall versus Krankheit ist in den letzten Jahren sprunghaft gestiegen, dies sowohl im Sozialversicherungsbereich (Unfall-, Invaliden-, Militärversicherung) wie auch im Bereich der Privatversicherungen (insbesondere Personenschäden im Motorfahrzeugbereich, private Unfallversicherung, ärztliche Haftpflicht usw.). Gleichzeitig werden an den medizinischen Gutachter heute wesentlich höhere Anforderungen gestellt als noch vor wenigen Jahren. Der üblicherweise in diagnostisch-therapeutischen Kategorien denkende Arzt sieht sich in der Rolle als Gutachter einem Auftraggeber gegenüber, der einem normativen «Ja-Nein»-Denken verpflichtet ist und der die in der medizinischen Wissenschaft öfter vorkommenden empirischen Unschärfen meist nur ungenügend nachzuvollziehen vermag. Die verschiedenen Denkansätze sind aber nur einer der Gründe, weshalb viele Gutachten aus Sicht der Administration und Justiz qualitativ oft nicht zu genügen vermögen.

Für den Beweiswert eines Gutachtens gelten folgende juristische Qualitätsanforderungen [1]: Das Gutachten soll für die streitigen Belange umfassend sein, es soll auf allseitige Untersuchungen beruhen, die beklagten Beschwerden berücksichtigen, es soll in voller Kenntnis der Vorakten abgegeben werden, die klinischen Zusammenhänge klar darlegen, Unklarheiten, die die Beantwortung gewisser Fragen erschweren oder verunmöglichen, deutlich machen. Die medizinische Interpretation wie die Schlussfolgerungen sollen begründet, einleuchtend und nachvollziehbar sein. Diverses kann den Aussagewert eines Gutachtens beeinträchtigen oder dieses für die administrativen Belange als ungenügend erscheinen lassen: nicht selten sind es ungenügende versicherungsrechtliche Terminologiekenntnisse des Gutachters, häufiger fehlende Sorgfalt in der kritischen Darlegung und Beurteilung der medizinischen Fakten, ungenügende Offenlegung von medizinischen Diskrepanzen und bleibenden Unklarheiten, man- gelhafte Erfassung und Bewertung der Vorakten, ungenügende Begründungen. Die Liste liesse sich beliebig verlängern. 1998 hat Meine [2] 262 Gutachten aus dem Privatversicherungsbereich qualifiziert, wobei er 35\% als einwandfrei taxierte, $36 \%$ als lückenhaft und $29 \%$ als schwer mangelhaft. Eine aktuelle SUVA-interne Bewertung von 102 Gutachten [3] ergab ein ebenso düsteres Bild. Die Ärzteschaft muss die Ausbildung und Qualitätssicherung von medizinischen Gutachtern zwingend selbst an die Hand nehmen [4], andernfalls werden sich externe Gremien darum bemühen [5]. Selbst im Bundesparlament wurde die Problematik im Dezember 2005 mittels einer Motion eingebracht [6]. Nur ganz wenige Fachgesellschaften haben die Aufgabe erkannt, die sie hier wahrzunehmen haben. Bisher hat lediglich die psychiatrische Fachgesellschaft bzw. die ihr angegliederte Gesellschaft für Versicherungspsychiatrie Leitlinien für Gutachten erstellt [7].

Der zunehmende Mangel an qualifizierten Gutachtern [8] bewog 1998 die SUVA, in Zusammenarbeit mit dem Schweizerischen Versicherungsverband SVV einen zweitägigen ärztlichen Gutachterkurs im Bereich der Unfallversicherung durchzuführen, der 1999 wiederholt wurde. Die spezielle Kursstruktur mit vielen Referenten aus dem direkt betroffenen Versicherungsbereich führte zum Vorwurf des Interessenkonfliktes, so dass die FMH, die Bedeutung dieser Kurse erkennend, im Jahr 2000 das Patronat übernahm und Prof. R. Kissling (Rheumatologe, Universitätsklinik Balgrist) zum Kursdirektor ernannte. Bis heute wurden in der Deutschschweiz fünf Grundkurse durchgeführt, die sich hauptsächlich auf den UVG-Bereich bezogen. Zwei identische Kurse wurden unter Kursleitung von Prof. R. Darioli, Lausanne, in der französischen Schweiz durchgeführt. 2004 fand der erste Folgekurs über «Begutachtungsprobleme im Grenzbereich von Psyche und Soma im Rahmen des UVG» statt, der im Dezember 2005 wiederholt wurde. Bisher haben über 400 Ärzte den Grundkurs und über 130 den Folgekurs besucht.

2003 wurde die Swiss Insurance Medicine SIM gegründet, eine Dachorganisation, die sich die Qualitätshebung der Versicherungsmedizin auf 
die Fahne geschrieben hat, bestehend aus Ärztinnen und Ärzten, die in verschiedenen Funktionen in der Versicherungsmedizin tätig sind (unter anderem Gutachter jeglicher Fachrichtung, Vertrauensärzte der Krankenkassen, RADÄrzte der IV, beratende Ärzte der Privatversicherer, Ärztinnen und Ärzte der Suva und der MV, aber auch an der Versicherungsmedizin interessierte Fachgesellschaften).

2004 wurde die SIM von seiten der FMH mit der Durchführung weiterer Kurse beauftragt, dies unter Beibehaltung des FMH-Patronats. Der schon lange bestehende Wunsch nach einer Ausdehnung der Kurse auf alle Versicherungszweige (neben UV auch IV, MV, KV, Privatversicherung, Haftpflicht) führte zur Bildung einer internen Arbeitsgruppe, die ein neues, modular aufgebautes Kurskonzept erarbeitete, das nach einem breiten Vernehmlassungsverfahren modifiziert wurde und nun realisiert werden soll.

\section{Neu strukturierte Gutachterausbildung}

Konkret gliedert sich die zukünftige modulare Gutachterausbildung, die mit einem Zertifikat abschliesst, in vier Kurse zu je 2 Tagen.

- Das Modul 1 bezieht sich vorwiegend auf die rechtlichen Aspekte der Versicherungsmedizin in bezug auf die verschiedenen Versicherungszweige, auf die versicherungstechnischen Termini und auf die vorwiegend technischen Aspekte der Gutachtenerstellung, wobei Magistralvorlesungen durch diverse Workshops ergänzt werden.

- Das Modul 2 befasst sich mit den besonderen Aspekten der Begutachtung (Gesprächsführung, Umgang mit den Parteien, spezielle juristische Aspekte usw.), wobei der zweite Tag dem Thema Psyche und Soma gewidmet ist, dies in Anlehnung an die beiden bereits durchgeführten Folgekurse nach altem System.

- Die Module 3 und 4 sind fachspezifische Kurse, die in enger Zusammenarbeit mit den diesbezüglich betroffenen und interessierten Fachgesellschaften durchgeführt werden sollen. Vorerst sind lediglich vier fachspezifische Schwerpunkte vorgesehen: Begutachtung «Allgemein», Begutachtung «Bewegungsapparat», Begutachtung «Neurologie», ebenso «Psychiatrie». Häufige fachbezogene Themen und Problemstellungen sollen in diesen Kursen mit erfahrenen Fachkollegen und Experten bearbeitet und besprochen werden.

- Das Modul 4 widmet sich zudem dem Kunstfehlergutachten sowie den häufigsten Gutachterfehlern.

\section{Zur Anerkennung der bisherigen Kurse:} Die seit 1998 durchgeführten Grundkurse werden im Rahmen des neuen Konzeptes als Modul 1 und die absolvierten Folgekurse als Modul 2 anerkannt, so dass Kolleginnen und Kollegen, welche solche Kurse nach altem System bereits absolviert haben, zwecks Erlangung des Zertifikates nur noch die fehlenden Module (eventuell 2, zudem 3 und 4) zu absolvieren haben.

\section{Kursdaten}

Modul 1:

17.-18. August 2006 (geschlossener Kurs, ausschliesslich für RAD-Ärzte der IV)

Modul 1:

11.-12. Januar 2007 (wird im August 2006 ausgeschrieben)

Modul 2:

November 2007

Modul 3:

voraussichtlich Mai 2007

Modul 4:

voraussichtlich März 2008

Kurse in französischer Sprache werden voraussichtlich 2007 beginnen.

Die Module 1-3 können chronologisch frei gewählt werden, das Modul 4 entspricht in jedem Fall dem Schlussmodul.

Nach Absolvierung aller vier Module wird ein SIM-Gutachterzertifikat erteilt. Voraussetzung zur Erlangung dieses Zertifikates ist ein Facharzttitel, die Absolvierung aller vier Module, die Vorlage eines eigenständig erstellten Gutachtens (6 Monate vor dem 4. Modul abzuliefern), das durch eine Expertengruppe (erfahrener Gutachter identischer Fachrichtung, Richter Versicherungsgericht, Versicherungsexperte) beurteilt wird. Die Expertenqualifikation des Gutachtens hat keinen sanktionierenden Effekt, es geht lediglich darum, dem Kursabsolventen die Möglichkeit zu geben, sein Gutachten zwecks eigener persönlicher Qualitätskontrolle kritisch beurteilen zu lassen.

Ängste vor sanktionierenden Auswirkungen dieser Zertifikate halten wir für unbegründet. Die Zertifikate bestätigen lediglich, dass der Betreffende eine entsprechende Schulung durchgemacht hat und damit über gewisse Grundkenntnisse verfügt, sie sagen aber nichts aus über die tatsächliche Qualifikation als Gutachter. Diesbezüglich wird auch zukünftig der freie 
Markt spielen: Der gute und neutrale Gutachter, der umfassend und ausgewogen in Kenntnis des aktuellen medizinischen Wissensstandes seine Expertise erstellt, wird immer begehrt sein.

Diese neuen modularen SIM-Gutachterkurse stehen weiterhin unter dem Patronat der FMH, zudem wird für jeden Kurs das «FMH-approved» eingeholt werden.

\section{Literatur}

1 BVE 122 V 160 Erw. 1c inkl. Hinweise.

2 Meine J. Ärztliche Begutachtung in der Schweiz Erfüllt sie die heutigen Qualitätsanforderungen? Swiss Surgery 1998;4:53-7.

3 Ludwig CA. Qualität fachärztlicher Gutachten im Bereich der sozialen Unfallversicherung. Medizinische Mitteilung SUVA Nr. 77/2006 (Publikation Juni 2006).

4 Soltermann B. Aus-, Weiter- und Fortbildung in Versicherungsmedizin und für die Festlegung der Arbeitsunfähigkeit. Schweiz Ärztezeitung 2004;85(38):2042-4.

5 Laeri MJ. Die Zertifizierung von Gerichtsexperten, ein Schweizer Novum; sasForum 2003;3:6-7.

6 Humbel Näf R. Motion 05.3905 an den Bundesrat: ATSG. Regelung der medizinischen Begutachtung, eingereicht am 16.12.2005.

7 Leitlinien der Schweizerischen Gesellschaft für Versicherungspsychiatrie für die Begutachtung psychischer Störungen. Schweiz Ärztezeitung 2004;85(20):1048-51.

8 Meine J. Gutachternotstand in der Schweiz. Schweiz Ärztezeitung 1998;79(7):240-1.

\section{Bisherige Gutachterkurse mit Schwergewicht UVG}

\section{Bisherige Kurse in Deutsch}

09/98 Grundkurs für ärztliche Begutachtung im Bereich Unfallversicherung (SUVA/ SVV)

09/99 Grundkurs für ärztliche Begutachtung im Bereich Unfallversicherung (SUVA/ SVV)

09/01 Grundkurs (ab 2001 unter FMH: Kursleitung Prof. R. Kissling, Balgrist)

09/02 Grundkurs

05/05 Grundkurs

12/04 Folgekurs Begutachtungsprobleme im Grenzbereich Psyche und Soma

12/05 Folgekurs Begutachtungsprobleme im Grenzbereich Psyche und Soma

Bisherige Kurse in Französisch:

05/00 Grundkurs (Kursleitung: Prof. R. Darioli, Lausanne)

09/03 Grundkurs

\section{Zukünftige SIM-Gutachterkurse}

\section{Modul 1}

Basiskurs Medizinische Gutachten [2 Tage]

- Der Gesundheitsschaden aus juristischer Sicht

- Kurzübersicht UV, MV, IV, KV, Privatversicherung, Haftpflicht

- Arbeitsunfähigkeit, Erwerbsunfähigkeit, Verweistätigkeit, Kausalität. Invalidität, Integritätsschaden, ATSG, die Schadenminderungspflicht und ihre medizinischen Konsequenzen (aus medizinischer, versicherungsrechtlicher und juristischer Sicht sowie bezogen auf die verschiedenen Versicherungen)

- Epidemiologie

- Rechtliche Aspekte des Gutachtens/Gutachters

- Aufbau eines Gutachtens

- Anforderungen an Gutachter, Ethik des Gutachters

- Wertigkeit klinischer Befunde/Sicherung der Diagnosen

- Einbezug psychosozialer und soziokultureller Faktoren in die gutachterliche Beurteilung

- Datenschutz und Gutachten

- Diverse Workshops zu versicherungsrechtlichen Aspekten in bezug auf die verschiedenen Versicherungen

- Folgende Punkte werden innerhalb der Themen zudem berücksichtigt: Bewertung von Observationsberichten; Beweiswert des Aktengutachtens; Anwesenheit des Anwaltes oder von Angehörigen; Rolle des Gutachters bei der Sachverhaltsermittlung; funktionelle Belastbarkeit und Haushaltsführung; notwendige zu liefernde Unterlagen (AVB); le barème médicale européen.

\section{Modul 2}

a) Die Begutachtung;

b) Psyche und Soma [2 Tage]

- Gesprächsführung durch Gutachter

- Der Umgang mit Exploranden, Versicherern, Auftraggebern

- Der Gutachter im Spannungsfeld der Parteien

- Häufige Verständigungsprobleme zwischen Medizinern und Juristen

- Physiologie und Psychologie des Schmerzes

- Krankheitsbilder/Diagnosen im Grenzgebiet zwischen Psyche und Soma

- Was ist eine psychische Krankheit? Zur Bedeutung nosologischer Modelle für die Begutachtung

- Rückenschmerz und Psyche

- Unterschiedliche Fragestellungen im Grenzgebiet Psyche/Soma bezüglich der verschiedenen Versicherungsarten 
Betrifft Fachgesellschaften, die sehr häufig mit Gutachten konfrontiert sind.
- Juristische Aspekte zum Grenzgebiet Psyche/ Soma

- Workshops zur gutachterlichen Gesprächsführung, zum Umgang mit den Parteien, zu Rücken und Psyche.

\section{Modul 3}

Fachspezifischer medizinischer Gutachterkurs I [2 Tage]

(unter Mitarbeit der Fachgesellschaften*)

- Folgende vier Schwerpunkte werden parallel angeboten:

1. Allgemein

2. Bewegungsapparat

3. Neurologie

4. Psychiatrie

(Wahl des Kurses nach Facharzttitel bzw. Interessengebiet)

- Magistralvorlesungen:

- fachübergreifende Themen

- Stellenwert von Testuntersuchungen

- die interdisziplinären Gutachten (Stellenwert, Problematik, Indikation, Methodik)

- In fachspezifischen / schwerpunktmässigen Workshops
- häufige fachspezifische Fälle

- fachspezifische Problemkreise

- juristische Aspekte zu fachspezifischen Fragen.

\section{Modul 4}

Fachspezifischer medizinischer Gutachter-

kurs II [2 Tage]

(unter Mitarbeit der Fachgesellschaften*)

- Schwerpunktmässige Workshops wie in Modul 3

- Stellenwert biomechanischer Beurteilungen und unfallanalytischer Gutachten

- Haftpflichtbegutachtung bei Kunstfehlern

- Die häufigsten Fehler in Gutachten - aus medizinischer Sicht

- aus versicherungsrechtlicher / juristischer Sicht

- Persönliche Besprechung des abgelieferten Gutachtens mit den beurteilenden Experten oder Aushändigung einer schriftlichen Beurteilung

- Aushändigung des SIM-Gutachterzertifikates. 